\title{
Dynamic Response Analysis of Tilting Pad Journal Bearing Considering Fluid-Structure Interaction
}

\author{
Q. Li ${ }^{\dagger}$, X. H. Tang, S. Zhang, Y. J. Wang, W. W. Xu and Z. B. Wang \\ College of New Energy, China University of Petroleum (East China), Qingdao 266580, China \\ $\dagger$ Corresponding Author Email: liqiangsydx@163.com
}

(Received December 18, 2020; accepted June 21, 2021)

\begin{abstract}
The transient hydrodynamic lubrication model of tilting pad journal bearings (TPJBs) was established by the computational fluid dynamics (CFD) method and the self-developed dynamic grid program. The fluid-structure interaction between the flow field and the rotor motion, the pads rotations was realized. The feasibility of the model is proved by comparing with the experimental data. The dynamic response of TPJBs under the various unbalance, the loading modes and the rotating speeds was studied. The dynamic response of TPJBs is further analyzed through a research of the relationships among the shaft whirl orbits, transient force acting on the shaft, rotation angles of the pads and transient oil film force of the pads. With the increase of unbalance, the whirl orbits expand and whirl orbits centers rise continuously. The whirl orbits and orbit center attitude angles of TPJBs are smaller than those of fixed-pad journal bearings. Compare with the load between pads, the whirl orbits are smaller and whirl orbits centers drop slightly under the load on pads. With the increase of rotating speed, the whirl orbits expand nonlinearly, whirl orbit center rises nonlinearly. The transient force acting on the shaft, the rotation angles of the pads and the transient oil film force of the pads change periodically, and the period and frequency of these changes are the same as that of the shaft rotation. The maximum force acting on the shaft appear before the maximum shaft center position (the vertexes of the whirl orbit).
\end{abstract}

Keywords: Tilting pad journal bearings; Transient model; Fluid-structure interaction; Dynamic response; Computational Fluid Dynamic (CFD); Dynamic grid.

\section{NOMENCLATURE}

$\begin{array}{ll}C_{c}, C_{e} & \text { empirical constants } \\ e & \text { eccentricity distance } \\ \vec{F} & \text { body force } \\ F_{x} & \text { horizontal oil film force components } \\ F_{y} & \text { vertical oil film force components } \\ f_{g} & \text { noncondensable gases mass fraction } \\ f_{k} & \text { vapor mass fraction of phase } \mathrm{k} \\ f_{v} & \text { vapor mass fraction } \\ f_{x} & \text { horizontal component of periodic load } \\ f_{y} & \text { vertical component of periodic load } \\ J_{o p} & \text { moment of inertia of the pad to the pivot } \\ M & \text { mass of the rotor } \\ M_{o p}\left(F_{p}\right) & \text { resultant moment of the pad } \\ n & \text { number of phases } \\ p_{v} & \text { liquid saturation vapor pressure }\end{array}$

$\begin{array}{ll}R_{c} & \text { vapor condensation rate } \\ R_{e} & \text { vapor generation rate } \\ \vec{v}_{d r, k} & \text { vapor generation rate } \\ \vec{v}_{k} & \text { velocity of phase } \mathrm{k} \\ \vec{v}_{m} & \text { mass averaged velocity } \\ \alpha_{k} & \text { volume fraction of phase } \mathrm{k} \\ \mu_{m} & \text { mixture viscosity } \\ \rho_{k} & \text { density of phase } \mathrm{k} \\ \rho_{l} & \text { liquid density } \\ \rho_{m} & \text { density of the mixture } \\ \rho_{v} & \text { vapor density } \\ \sigma & \text { surface tension coefficient of the } \\ & \text { liquid } \\ \omega & \text { rotating angular velocity } \\ \omega_{p} & \text { angular velocity of the pad }\end{array}$




\section{INTRODUCTION}

Hydrodynamic journal bearings are widely used in rotating machinery, such as steam turbines and compressors. The rotating machinery is becoming larger in scale with higher speeds as its efficiency and economy improved. Compared with the fixedpad journal bearings, the tilting pad journal bearings (TPJBs) have higher stability and reliability, so the TPJBs are more suitable for large-scale and highspeed rotating machinery. Due to the manufacturing and assembly error, there is unbalance in the rotor system, which affects the dynamic response of TPJBs. Moreover, the structural characteristics of TPJBs, such as the pads, make the dynamic response of TPJBs more complex. Therefore, it is necessary to study the dynamic response of TPJBs under the unbalance.

The hydrodynamic lubrication of journal bearings was first discovered by Tower, and then the basic lubrication equation (Reynolds equation) was proposed by Reynolds through theoretical analysis (Tower 1983; Reynolds 1886). The numerical solution of the Reynolds equation is obtained by the finite difference method in order to study the performances of journal bearings. El Kayar (1983) and $\mathrm{Li}$ et al. (2000) used the finite difference method to solve the two-dimensional Reynolds equation to further study the influence of the eccentricity, rotation speed and the characteristics of the lubricating medium on the fixed-pad journal bearings performance. Zheng and Hasebe (2000) calculated the static equilibrium position and dynamic characteristic coefficients of the fixedpad journal bearings based on the Reynolds equation considering the effect of cavitation. Roy and Laha (2009) also solved the Reynolds equation via the finite difference method, studied the fixedpad journal bearing with an oil groove on the pad, and analyzed the influence of the angle and the length of the oil groove on the static and dynamic characteristics of the bearings. Lahmar and BouSaid (2008) studied the effects of coupling stress on the dynamic behavior of connecting rod bearings bearing by solving the modified Reynolds equation.

In addition, the TPJBs were also studied based on Reynolds equation. The Reynolds equation and energy equation were solved by the finite difference method in order to study the influence of the lubricant viscosity, heat conduction and deformation on the TPJBs, the influence of the inlet pressure on the thermohydrodynamic performance of four-pads TPJBs (Taniguchi et al. 1990; Ha et al. 1994). Wang and Liu (2011) further solved the generalized Reynolds equation and the moment balance equation and studied the relationship between the rotation angle of the pads, the eccentricity and the clearance rate at the static equilibrium position of TPJBs. Varela et al. (2013) studied the steady-state performances of TPJBs under the condition of controllable lubrication by solving Reynolds equation. Raud et al. (2013) researched effects of lift pockets on the TPJBs performance based on Reynolds equation.
With the development of computer technology, it is possible to solve Navier-Stokes (N-S) equations. Based on the computational fluid dynamics (CFD) method, the three-dimensional (3D) lubrication flow field of journal bearings has been researched. Gertzos et al. (2008) conducted a CFD analysis of journal bearings with a Bingham lubricant. Li et al. (2012 a, b) presented a 3D transient model of journal bearings and combined the CFD method with the Fluid-Structure Interaction (FSI) method in order to study the transient lubrication flow field of a journal bearing-rotor system.

Compared with the fixed-pad journal bearings, the motion of TPJBs is more complicated, including the movement of shaft and the rotation of each pad. Tong and Palazzolo (2018) then studied the influence of journal eccentricity, oil supply temperature and rotational speed on the temperature distribution of TPJBs considering the Morton effect via experiments and numerical simulations. Ding et al. (2018) investigated the friction loss of TPJBs considering air entrainment and the cavitation effect based on CFD method, analyzed the gas-liquid distribution in the bearing clearance and the cause of friction loss, and proposed the improvement method. Parovay and Falaleev (2017) researched the performance of elastically supported TPJBs under oil-deficient conditions and determined the relationship between the performances of TPJBs and the load, speed and radial clearance. Armentrout et al. (2017) analyzed the influence of turbulence and convective inertia on water-lubricated TPJBs using the traditional Reynolds equation method and the CFD method, respectively. The results showed that the turbulence term improves the bearing capacity by $30-50 \%$. Marx et al. (2018 a, b) studied the phenomenon of viscosity modifiers thinning under a strong shear stress, and the effect of lubricant shear thinning on the performance of TPJBs. Lee et al. (2018) conducted a thermohydrodynamic analysis and experiments on TPJBs under wear conditions and studied the effect of the wear depth on the surface pressure and temperature of bearing pads. Yang and Palazzolo (2021a, b) further researched the thermo-elasto hydrodynamic mixing prediction model of TPJBs based on CFD method

In summary, several studies have been conducted on bearing performances based on the Reynolds equation and CFD method, but most of them are steady-state bearing performances, with few studies on dynamic response performances of bearings. In this paper, the 3D transient fluid lubrication model of TPJBs is established by using the CFD method and a self-developed dynamic grid method. The influence of the unbalance, the loading modes and the rotating speed on the dynamic response of the TPJBs is studied. The relationships among the whirl orbits, the transient force acting on the shaft, rotation angles of the pads and the transient oil film force of the pads are further analyzed. 


\section{Model AND Methods}

\subsection{Physical Model}

The research object of this paper is five-pad TPJBs. To reduce the number of grids and improve the calculation speed, a half-model is constructed according to the symmetry of the flow field and the TPJB structure. Radial oil supply is adopted. The oil inlet is set on the back of the pad in order to eliminate the influence of the inlet impact on the bearing oil film. The lubricant enters from the oil inlet, passes through the annular oil path and the clearance between the pads, enters the bearing clearance, and finally flows out from the clearance between the two sides of the bearing. According to load directions, there are two main loading modes, the load between pads (LBP) and the load on pad (LOP) (Lai et al. 2014). The 3D watershed model and the boundary conditions of five-pad TPJBs are shown in Fig. 1.

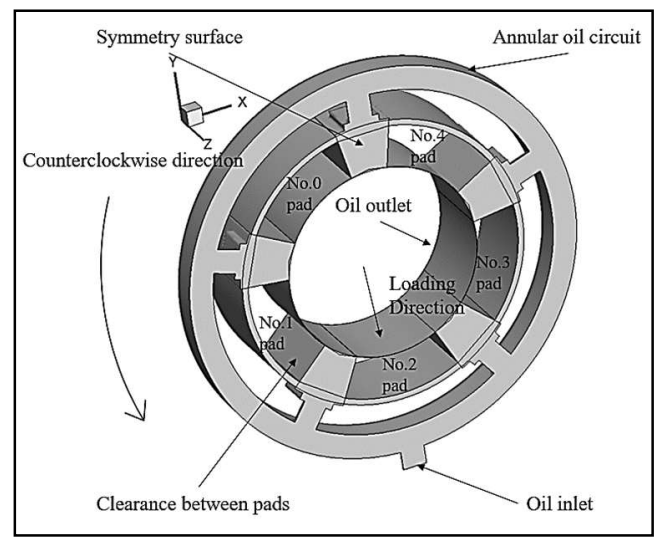

Fig. 1. 3D watershedmodel and the boundary conditions of TPJBs.

The \#32 steam turbine oil is selected as the lubricant. The specific size of the TPJBs and the parameters of the \#32 steam turbine oil are listed in Table 1.

Table 1 Size of the TPJBs and the parameters of the \#32 steam turbine oil.

\begin{tabular}{|c|c|c|c|}
\hline \multirow{4}{*}{ Bearing } & Properties & Unit & Value \\
\cline { 2 - 4 } & Bearing radius & $\mathrm{mm}$ & 16 \\
\cline { 2 - 4 } & Bearing length & $\mathrm{mm}$ & 29.5 \\
\cline { 2 - 4 } & Radius clearance & $\mathrm{mm}$ & 0.032 \\
\cline { 2 - 4 } & Number of pads & - & 5 \\
\cline { 2 - 4 } & Pad arc angle & $\mathrm{deg}$ & 50 \\
\cline { 2 - 4 } & Pad length & $\mathrm{mm}$ & 7 \\
\cline { 2 - 4 } & Pad offset & - & 0.5 \\
\cline { 2 - 4 } & Density of material & $\mathrm{kg} /$ & 7930 \\
& & \\
\hline \multirow{7}{*}{$\# 32$ oil } & Specific heat & $\mathrm{J} / \mathrm{kg}$ & 500 \\
& Lubacity of material & $\cdot \mathrm{K}$ & \\
\cline { 2 - 4 } & Lubricant viscosity & $\mathrm{Pa} \cdot \mathrm{s}$ & 0.022 \\
\cline { 2 - 4 } & Lubricant density & $\mathrm{kg} /$ & 850 \\
& & $\mathrm{~m}^{3}$ & \\
\hline
\end{tabular}

\subsection{Governing Equation}

The three-dimensional flow field of TPJBs was calculated using the CFD method. There are gas and liquid lubricant in the TPJBs when cavitation is taken into consideration. This multiphase flow is described by the "Mixture" model. This paper focuses on the establishment of transient model of TPJBs, and then realizes the fluid-structure interaction between the transient flow field and the rotor motion, pads rotations, the influence of temperature and shear on the viscosity of lubricant is not considered.

$\frac{\partial \rho_{m}}{\partial t}+\nabla \mathrm{g}\left(\rho_{m} v_{m} \underset{\mathrm{r}}{\mathrm{r}}\right)=0$

where $\vec{v}_{m}$ is the mass averaged velocity; $\rho_{m}$ is the density of the mixture.

The momentum equation is given as:

$$
\begin{aligned}
& \frac{\partial}{\partial t}\left(\underset{\rho_{m}}{\stackrel{\mathrm{r}}{v_{m}}}\right)+\nabla\left(\underset{\rho_{m}}{\stackrel{\mathrm{r}}{v_{m}}{ }^{\mathrm{r}} v_{m}}\right)=-\nabla p+\nabla\left[\mu_{m}\left(\begin{array}{c}
\nabla v_{m}^{\mathrm{r}}+ \\
\nabla \underset{v_{m}}{\mathrm{r}} T^{\prime}
\end{array}\right)\right]_{(2)} \\
& +\rho_{m} \stackrel{\mathrm{r}}{g}+\stackrel{\mathrm{r}}{F}+\nabla\left(\sum_{k=1}^{n} \alpha_{k} \rho_{k} v_{d r, k} v_{d r, k}\right)
\end{aligned}
$$

where $n$ is the number of phases; $\vec{F}$ is a body force; and $\mu_{m}$ is the mixture viscosity.

The mass transfer can be solved using the vapor transport equation given below:

$\frac{\partial}{\partial t}\left(\rho_{m} f_{k}\right)+\nabla\left(\rho_{m}{ }_{v_{m}}^{\mathrm{r}} f_{k}\right)=\nabla\left(\mu_{m} \nabla f_{k}\right)+R_{e}-R_{c}$

where $f_{k}$ is the vapor mass fraction of phase $\mathrm{k}$.

The mixture density and mass averaged velocity can then be calculated by Eq. (4) and Eq. (5), respectively:

$\underset{v_{m}}{\mathrm{r}}=\frac{\sum_{k=1}^{n} \alpha_{k} \rho_{k}{\stackrel{\mathrm{r}}{v_{k}}}_{\rho_{m}}}{\rho_{m}}$

$\rho_{m}=\sum_{k=1}^{n} \alpha_{k} \rho_{k}$

where $\alpha_{k}$ is given by the following Eq. (6):

$\alpha_{k} \equiv f_{k} \frac{\rho_{m}}{\rho_{v}}$

In the TPJBs, the clearance between journal and bearing is not uniform because of eccentricity of the shaft. Where the clearance is divergent, a lowpressure region is formed. When the oil enters the bearing divergence wedge and the oil film pressure drops below the saturation vapor pressure, the cavitation occurs and the gaseous lubricant is generated. The source term in Eq. (3) is calculated by the "full cavitation model" proposed by Singhal et al. (2002). The "full cavitation model" is based on the multiphase flow framework, which takes all the firstorder effects into account, and can deal with the large density changes related to the phase transition of the fluid without the need to determine the location, extent, or type of cavitation in advance (Li et al. 2017a). The "full cavitation model" can be described as follows Eq. (7) and Eq. (8): 


$$
R_{e}=C_{e} \frac{\sqrt{k}}{\sigma} \rho_{l} \rho_{v} \sqrt{\frac{2\left(p_{v}-p\right)}{3 \rho_{l}}}\left(1-f_{v}-f_{g}\right) \quad p<p_{v}
$$

$$
R_{c}=C_{c} \frac{\sqrt{k}}{\sigma} \rho_{l} \rho_{v} \sqrt{\frac{2\left(p-p_{v}\right)}{3 \rho_{l}}} f_{v} \quad p>p_{v}
$$

where $C_{e}$ and $C_{c}$ is the empirical constants; $\rho_{l}$ is the liquid density; $\rho_{v}$ is the vapor density; $\sigma$ is surface tension coefficient of the liquid; $f_{v}$ is the vapor mass fraction; $f_{g}$ is the noncondensable gases mass fraction; $p_{v}$ is the liquid saturation vapor pressure.

\subsection{Transient Model}

Under the action of the unbalance and the oil film force, the shaft whirls in the TPJBs. The whirl of the shaft is a transient process. Therefore, it is necessary to establish a transient model in order to simulate the whirl process of the shaft and then to study the dynamic response performances of the TPJBs. The different unbalance are realized by applying periodic loads with different amplitudes. The component of the periodic load in the horizontal and vertical directions is given by the following Eq. (9):

$$
\left\{\begin{array}{l}
f_{x}=M e \omega^{2} \cos (\omega t) \\
f_{y}=M e \omega^{2} \sin (\omega t)
\end{array}\right.
$$

where $M$ is the mass of the rotor; $e$ is the eccentricity distance; and $\omega$ is the rotating angular velocity.

The motion of the shaft in the bearing is determined by the force acting on the journal. The equation of motion of the journal is as Eq. (10):

$$
\left\{\begin{array}{l}
M=F_{x}+f_{x} \\
M=F_{y}-M g+f_{y}
\end{array}\right.
$$

where $F_{x}$ and $F_{y}$ are the horizontal and vertical oil film force components, respectively.

The oil film force component is obtained by integrating the surface pressure of the journal, and the different periodic loads are obtained by changing the unbalance eccentricity distance $e$.

During the whirling process of the shaft, each pad will swing correspondingly, which is one of the characteristics of TPJBs and the biggest difference from the fixed-pad bearings. Looking from the positive $\mathrm{z}$-axis, the counterclockwise rotations of the pads is defined as positive, and the clockwise rotation of the pads is defined as negative. The rotations of the pads around the pivot is determined by the moment acting on the pads, and the equation of motion of the pads can be obtained by the theorem of the momentum moment as follows:

$\frac{d}{d t} J_{o p} \omega_{p}=M_{o p}\left(F_{P}\right)$ where $J_{o p}$ is the moment of inertia of the pad to the pivot; $\omega_{p}$ is the angular velocity of the pad; and $M_{o p}\left(F_{p}\right)$ is the resultant moment on the pad.

The rotation moment of the pads are obtained by integrating the upper and lower surface pressure of the pads and then multiplying the distances to the pivot. The rotation angular velocity of the pad can be obtained from Eq. (11). The whirl of the shaft, the rotation of the pads and the precise movement of the grid nodes are then realized by the self-developed dynamic grid method on the CFD soft-ware FLUENT.

The calculation process is shown in Fig. 2. Firstly, the transient oil film force is obtained by integrating the surface pressure of the shaft, and then the displacement of the shaft under the current time step is calculated. Then, the surface pressure of each pads is integrated to calculate the rotation angles of each pad in the current time step. Finally, the movement of the shaft and the rotation of each pad are completed via user defined functions (UDFs) (Li et al. 2016). The dynamic grid update is completed in the current time step.

During the transient calculation process, the grid nodes are accurately moved, and a high grid quality is guaranteed. The grid deformation after dynamic grid updating is shown in Fig. 3. To better display the grid, the bearing clearance and pad rotation angle are artificially increased.

\subsection{Boundary Condition and Discrete Method}

The inlet and outlet conditions of the TPJBs are pressure boundary conditions, the inlet oil supply pressure was $0.3 \mathrm{MPa}$, and the outlet pressure was $0.1 \mathrm{MPa}$ (Li et al. 2019). The unbalance eccentricity distance were $30 \mu \mathrm{m}, 50 \mu \mathrm{m}, 80 \mu \mathrm{m}$ and $150 \mu \mathrm{m}$, respectively. The rotation speed then increased from $400 \mathrm{rad} / \mathrm{s}$ to $1600 \mathrm{rad} / \mathrm{s}$ every $200 \mathrm{rad} / \mathrm{s}$. The finite volume method was used to discretize the governing equation, and the SIMPLE scheme was used for pressure-velocity coupling. The PRESTO! was chosen for pressure interpolation due to the highspeed rotating flow in the TPJBs. It should be noted that the Reynolds number in all cases studied in this paper was less than 400 , and the laminar flow model was adopted.

\section{GRID INDEPENDENCE AND MODEL VALIDATION}

\subsection{Grid Independence}

The structured grids are used to partition the dynamic grid area in order to achieve an accurate movement of the grid nodes. The dynamic grid areas are the bearing clearance, the back areas of pad and the areas between the pads. The grid distribution is shown in Fig. 4. The sensitivity of the calculation results of the number of grids is checked for the bearing clearance domain of the TPJBs under an eccentricity of 0.4 The maximum pressure of the bearing clearance with different grid numbers is compared to ensure that the 


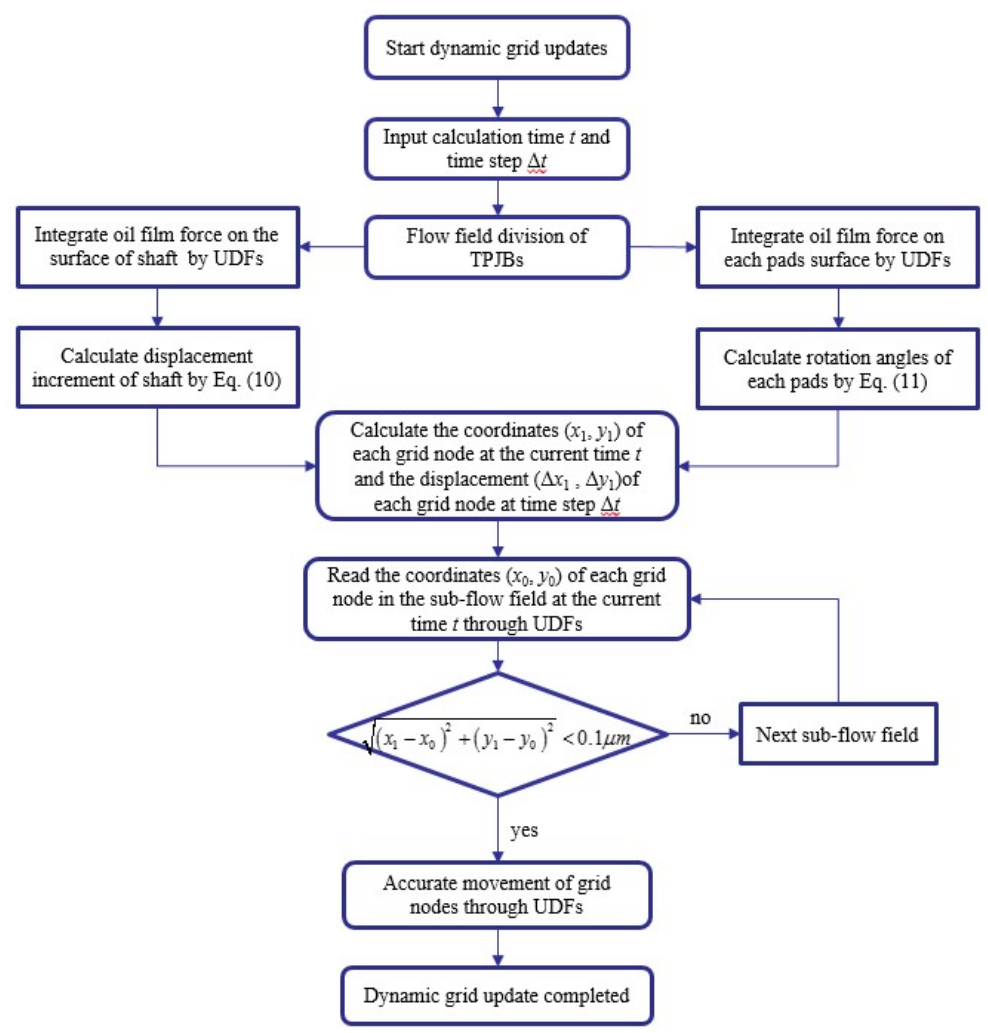

Fig. 2. Transient calculation process.

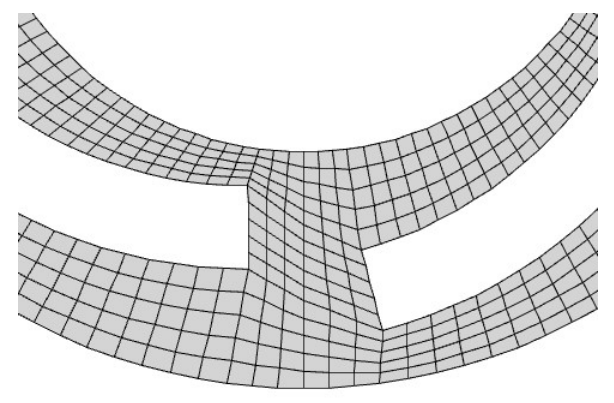

Fig. 3. Grid deformation after dynamic grid updating.

sensitivity of the grid numbers is sufficiently low. The deviation of the maximum pressure decreases with an increase in the grid layers and grid numbers, as shown in Table 2 . The deviation is $0.43 \%$ (less than $1 \%$ ), when the number of grids is 336,000 and the number of radial grid layers is 5 . Therefore, under the premise of ensuring the accuracy of calculation, the 5 radial divisions, 560 circumferential divisions and 40 axial divisions are adopted to reduce the calculation time.

Table 2 Grid independence calculation results

\begin{tabular}{|c|c|c|c|}
\hline $\begin{array}{c}\text { Grid } \\
\text { number }\end{array}$ & $\begin{array}{c}\text { Radial } \\
\text { grid } \\
\text { layers }\end{array}$ & $\begin{array}{c}\text { Maximum oil } \\
\text { film pressure } \\
(\mathrm{Pa})\end{array}$ & $\begin{array}{c}\text { Deviation } \\
(\%)\end{array}$ \\
\hline 201600 & 3 & 1742910 & 7.55 \\
\hline 268800 & 4 & 1839929 & 2.40 \\
\hline 336000 & 5 & 1877148 & 0.43 \\
\hline 403200 & 6 & 1885237 & reference \\
\hline
\end{tabular}

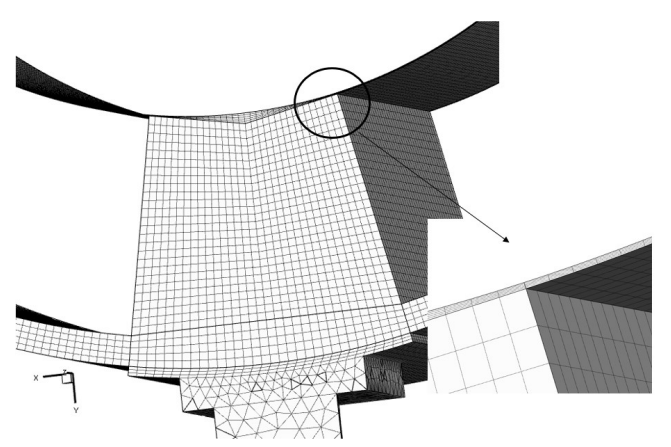

Fig. 4. Grid distribution.

\subsection{Model Validation}

To validate the $3 \mathrm{D}$ transient model developed in this paper, the simulated results are compared with the experimental data by Hagemannn et al. (2019) with same conditions listed in Table 3 . Under the action of bearing load and oil film force, the rotating shaft gradually moves from the initial position to the static equilibrium position. The above process is realized by self-made dynamic grid program, and the fluid-structure interaction between lubrication flow field, shaft and pads is ensured. Figure 5 shows the comparison between the simulation results and the experimental data. It can be seen that the simulation results of the circumferential oil film pressure agree well with the experimental data at the bearing mid-plane. Therefore, the transient model and the calculation model in this paper are feasible. 
Table 3 Specifications of the bearing and test conditions

\begin{tabular}{|c|c|c|c|}
\hline \multirow{7}{*}{ Bearing } & Properties & Unit & Value \\
\cline { 2 - 4 } & $\begin{array}{c}\text { Bearing } \\
\text { diameter }\end{array}$ & $\mathrm{mm}$ & 120 \\
\cline { 2 - 4 } & $\begin{array}{c}\text { Bearing } \\
\text { length }\end{array}$ & $\mathrm{mm}$ & 72 \\
\cline { 2 - 4 } & $\begin{array}{c}\text { Radius } \\
\text { clearance }\end{array}$ & $\mathrm{mm}$ & 0.108 \\
\cline { 2 - 4 } & $\begin{array}{c}\text { Number of } \\
\text { pads }\end{array}$ & - & 4 \\
\cline { 2 - 4 } conditions & Pad arc angle & $\mathrm{deg}$ & 70 \\
\cline { 2 - 4 } & Pad length & $\mathrm{mm}$ & 20 \\
\cline { 2 - 4 } & Pad offset & - & 0.6 \\
\cline { 2 - 4 } & Bearing load & $\mathrm{MPa}$ & 2 \\
\cline { 2 - 4 } & $\begin{array}{c}\text { Lubricant } \\
\text { flow rate }\end{array}$ & $1 / \mathrm{min}$ & 90 \\
\cline { 2 - 4 } & $\begin{array}{c}\text { Rotational } \\
\text { speed }\end{array}$ & $\mathrm{r} / \mathrm{min}$ & 12000 \\
\hline
\end{tabular}

Fig. 5. Comparison between the numerical results and experimental results.

\section{RESULTS AND DISCUSSION}

\subsection{Dynamic Responses of Different Unbalances}

To explore the dynamic response of the TPJBs, the influences of different unbalances are studied in this section. The rotational speed is $400 \mathrm{rad} / \mathrm{s}$ and the applied load (half the weight of the shaft) is 171.76 $\mathrm{N}$, which is the same as that in Li et al. (2019). The loading mode is the LOP.

The simulation results of the shaft whirl orbits of the TPJBs are compared with the fixed-pad journal bearings in $\mathrm{Li}$ et al. (2019) under different unbalances, as shown in Fig. 6. The maximum difference values of the shaft whirl orbit sizes of the TPJBs and the fixed-pad journal bearings in the $\mathrm{x}$ and $\mathrm{y}$ directions are shown in Table. 4 and the positions of shaft whirl centers are shown in Fig. 7, under different unbalance. With an increase of the unbalance, the shaft whirl orbits expand continuously, $\Delta \mathrm{x}$ from 5.757 to $23.471 \mu \mathrm{m}, \Delta \mathrm{y}$ from 4.612 to $21.985 \mu \mathrm{m}$. The centers of the shaft whirl orbits rise about $4 \mu \mathrm{m}$. This shows that the stability of the TPJBs is reduced. Compared with the fixed-pad bearings, the size of the shaft whirl orbits obviously decreases, which is more obvious in the $\mathrm{x}$ direction than in the $\mathrm{y}$ direction. The centers of the shaft whirl orbits of the TPJBs are near the yaxis, with smaller attitude angles, which is consistent with that in Li et al. (2017). The centers of the shaft whirl orbits of the fixed-pad journal bearings are on the right side of the y-axis, and the half-frequency whirl occurs in the fixed-pad bearing when the unbalances are $50 \mu \mathrm{m}$ and $80 \mu \mathrm{m}$. This shows that the stability of the TPJBs is higher than that of the fixed-pad journal bearings. However, in the y-axis direction, the shaft whirl orbits and whirl orbit center of the TPJBs obviously drop compared with the fixed-pad journal bearings.

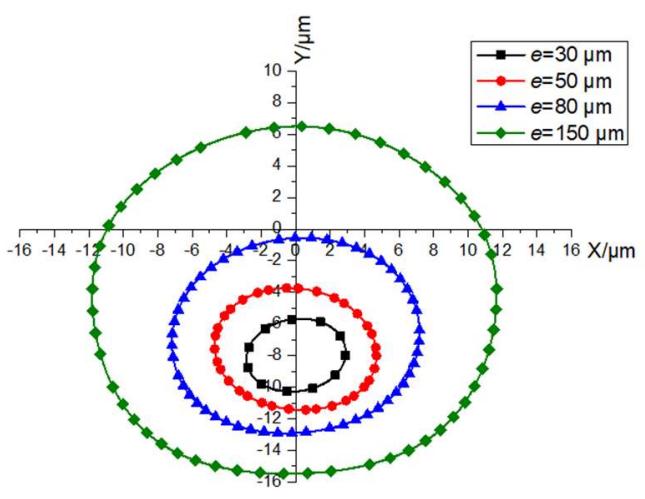

(a) Shaft whirl orbits of the TJPBs

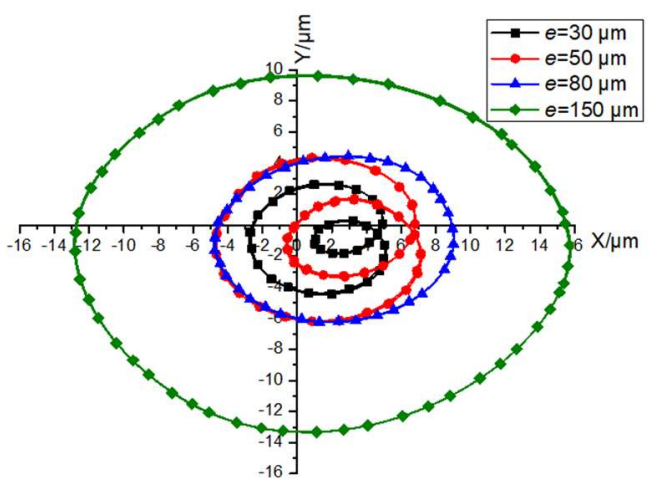

(b) Shaft whirl orbits of the fixed-pad journal bearing

Fig. 6. Shaft whirl orbit of the TPJBs and the fixed-pad journal bearing.

Table 4 Maximum differences in the shaft whirls of the TPJBs and fixed-pad journal bearings

\begin{tabular}{|c|c|c|c|c|}
\hline & \multicolumn{2}{|c|}{ TPJBs } & \multicolumn{2}{c|}{ Journal bearings } \\
\hline & $\Delta x$ & $\Delta y$ & $\Delta x$ & $\Delta y$ \\
\hline $\begin{array}{c}e=30 \\
\mu \mathrm{m}\end{array}$ & 5.757 & 4.612 & 7.885 & 7.161 \\
\hline $\begin{array}{c}e=50 \\
\mu \mathrm{m}\end{array}$ & 9.397 & 7.715 & 11.890 & 10.601 \\
\hline $\begin{array}{c}e=80 \\
\mu \mathrm{m}\end{array}$ & 14.370 & 12.352 & 13.850 & 10.711 \\
\hline $\begin{array}{c}e=150 \\
\mu \mathrm{m}\end{array}$ & 23.471 & 21.985 & 28.6433 & 22.996 \\
\hline
\end{tabular}




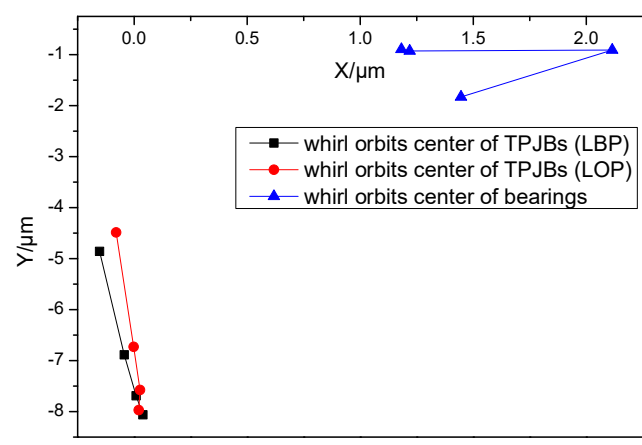

Fig. 7. Centers of the shaft whirl orbits of the TPJBs and fixed-pad journal bearings.

\subsection{Dynamic Responses of the Different Loading Mode and Rotational Speed}

To further study the dynamic response of TPJBs under the unbalances, the influences of the loading mode and rotating speed are considered. The shaft whirl orbits considering different loading modes (rotational speed: $400 \mathrm{rad} / \mathrm{s}$ ) and rotational speed (unbalance: $\mathrm{e}=30 \mu \mathrm{m}$ ) are shown in Figs. 8 and 9, respectively. The shaft whirl orbits enlarge, and the bearing stability decreases with an increase of unbalance under LBP, as shown in Fig. 8. Compared with LOP, the centers of the shaft whirl orbits drop slightly under LBP, as shown in Fig. 7. There is a gap between the load pads on both sides under LBP, which leads to the decrease of local oil film pressure. Therefore, greater eccentricity is needed to obtain enough oil film force. However, the shaft whirl orbits sizes of TPJBs are smaller under LBP, which is obvious at the lower side of the whirl orbits. This indicates that the LBP can provide more stability margins in the $\mathrm{x}$ direction, which improves the stability of the TPJBs. This is consistent with the conclusion in Lai et al. (2014).

With an increase in the rotational speed, the influences of the unbalance increase, the whirl orbits enlarge, and the center of whirl orbits rise but remain near the y-axis, as shown in Fig. 9. Under a rotational speed of 400-1600 rad/s, the maximum values of the whirl orbits are from $4 \mu \mathrm{m}$ to approximately $20 \mu \mathrm{m}$. This shows that even a small unbalance will have a greater impact on the dynamic response of the rotor system under a high-speed rotating state.

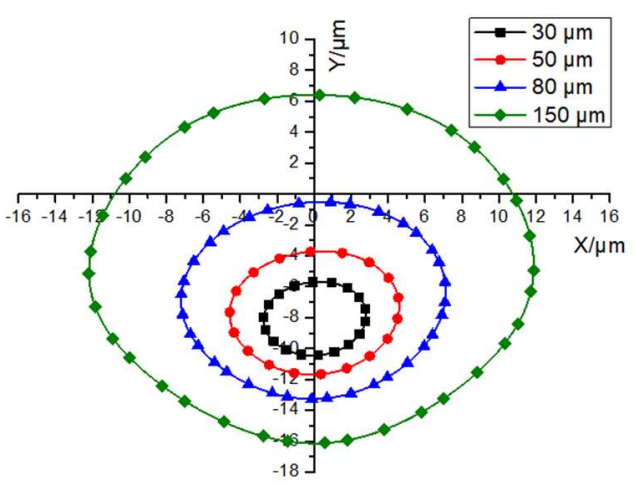

Fig. 8. Shaft whirl orbits for LBP.

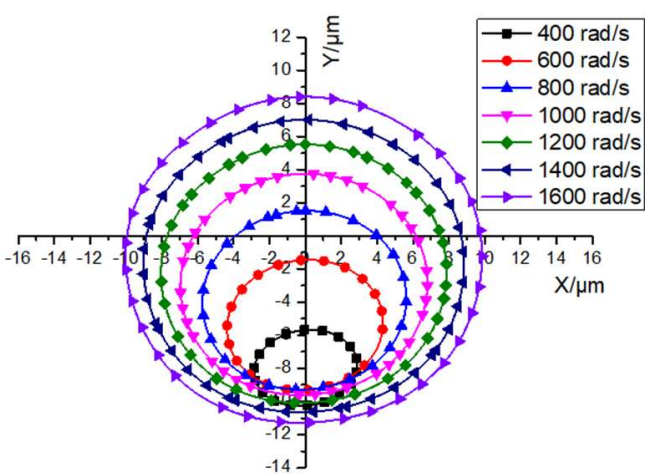

Fig. 9. Shaft whirl orbits of different rotational speeds.

\subsection{Dynamic Response Analysis of TPJBs}

The dynamic response of TPJBs is complex due to the increase in the degrees of freedom of the pads. The relationship between the shaft whirl orbit, the transient force, the rotation angle of each pad, and oil film force of each pad is discussed in this section in order to analysis the dynamic response of TPJBs. The unbalance is $30 \mu \mathrm{m}$ and the rotating speed of shaft is $800 \mathrm{rad} / \mathrm{s}$.

The positions of shaft center in the $\mathrm{x}$ and $\mathrm{y}$ direction and the transient force acting on the shaft in the $\mathrm{x}$ and $\mathrm{y}$ direction are shown in Figs. 10 . During the shaft whirling process, the positions of shaft center and the transient force acting on the shaft change with sine functions in $\mathrm{x}$ and $\mathrm{y}$ direction. The change period and the frequency of transient force and shaft center is $0.007854 \mathrm{~s}$ and $127.3239 \mathrm{~Hz}$, which is the same as the rotation frequency of shaft. There is phase difference between the change of shaft center position and the change of transient force in $\mathrm{x}$ and $\mathrm{y}$ direction. In one whirl period, the maximum transient force acting on the shaft in the $\mathrm{x}$ and $\mathrm{y}$ direction appears before the maximum position (the vertexes of the whirl orbit) of the shaft center in the $\mathrm{x}$ and $\mathrm{y}$ direction.

The shaft whirl orbit and the rotation angles of pads are shown in Figs.11. In Fig. 11(a), the starting position of the shaft whirl is marked with red circular dot. The whirl orbit is divided into left and right sides with the $\mathrm{x}$-axis as the boundary. The pad number is shown in Fig. 1. During the whirling process of the shaft, the rotation angle of each pad also changes harmonically, and the change period and frequency are the same as those of the shaft. However, the rotation direction and size of each pad are different. In one whirl period, when the shaft is on the right side of the whirl orbit, the pads No. 0 and No. 1 rotate counterclockwise first and then clockwise, and the pads No. 3 and No. 4 rotate clockwise first and then counterclockwise; when the shaft is on the left side of the whirling orbit, the pads No. 0 and No. 1 rotate clockwise first and then counterclockwise, and the pads No. 3 and No. 4 rotate counterclockwise first and then clockwise. 


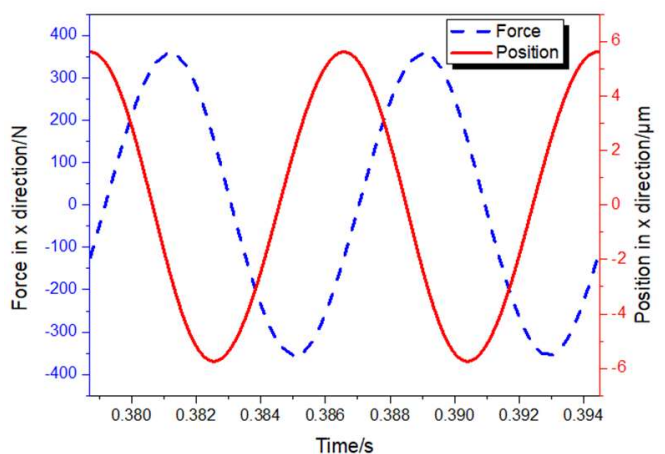

(a) Transient force and position of shaft in $\mathrm{x}$ direction

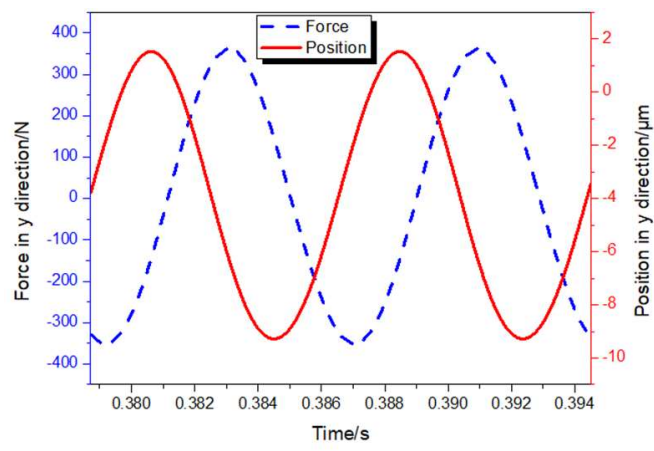

(b) Transient force and position of shaft in y direction

Fig. 10. Transient force and position of shaft in $x$ and $\mathbf{y}$ direction.

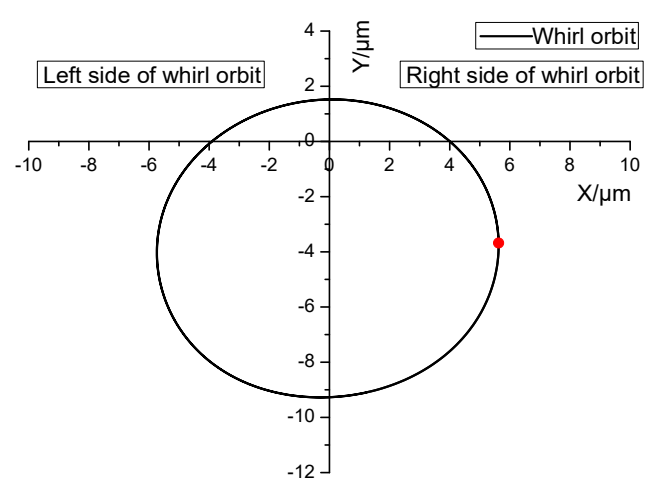

(a) Shaft whirl orbit

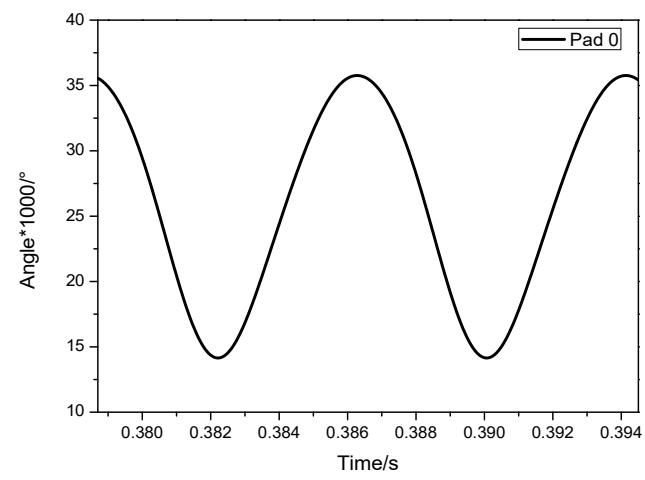

(b) Rotation angle of the pad 0

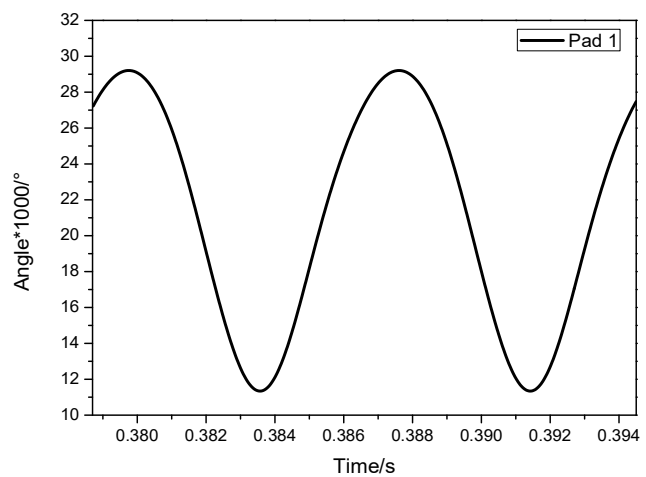

(c) Rotation angle of the pad 1

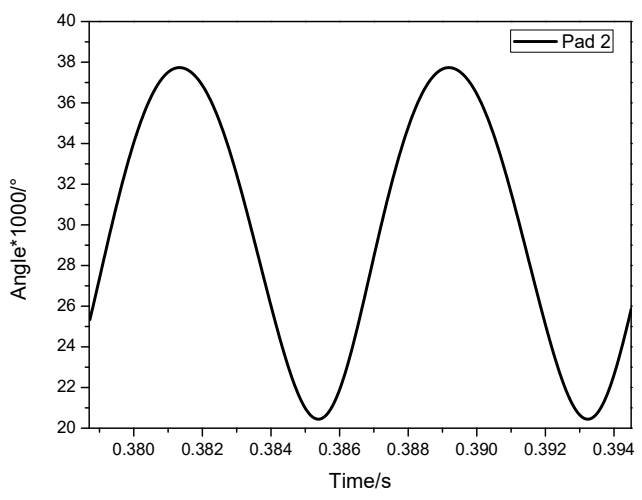

(d) Rotation angle of the pad 2

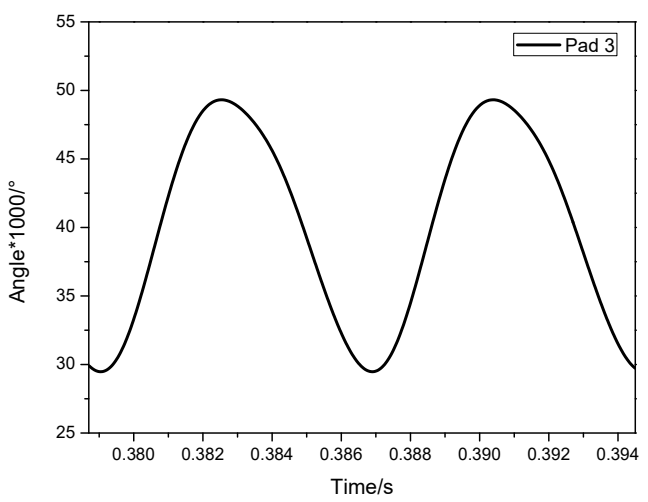

(e) Rotation angle of the pad 3

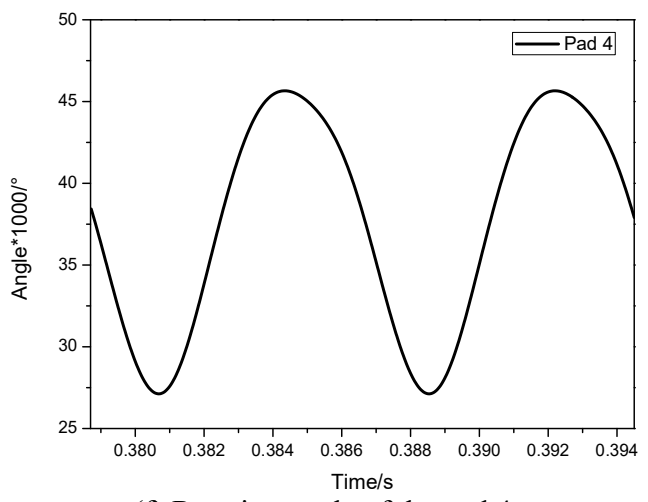

(f) Rotation angle of the pad 4

Fig. 11. Shaft whirl orbit and rotation angle of the pads. 


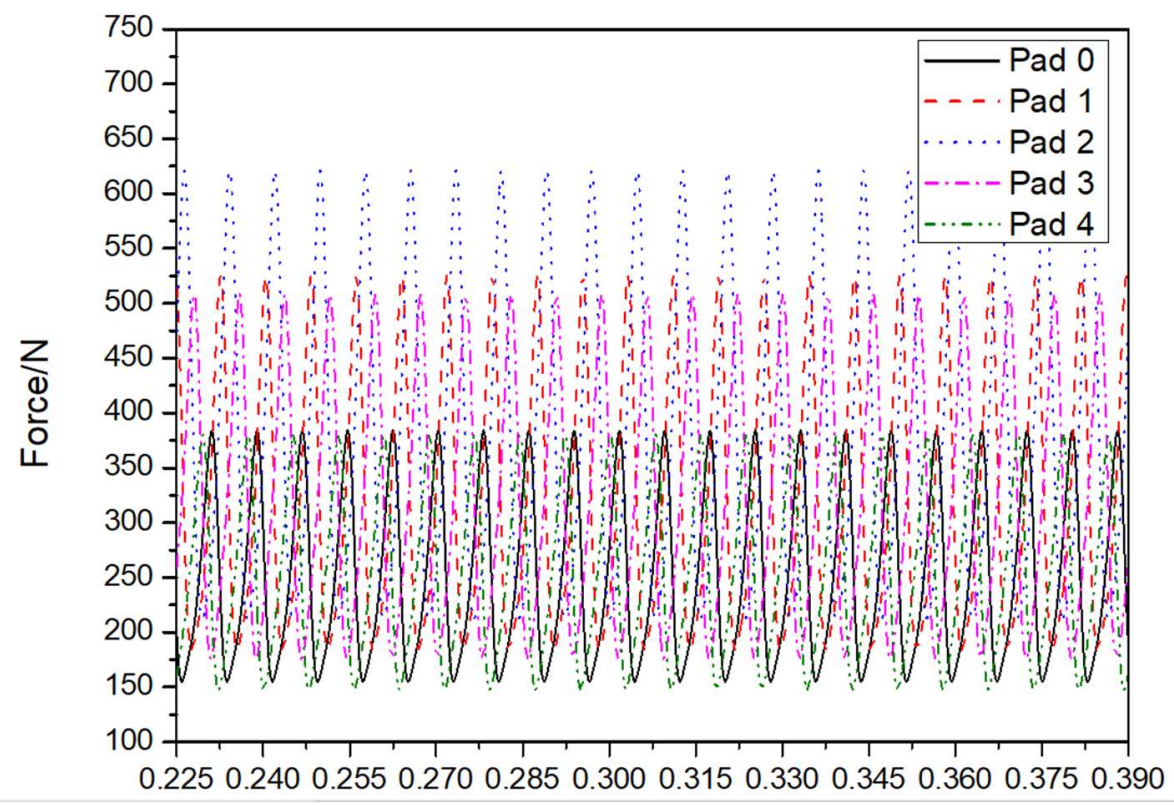

Fig. 12 Transient oil film force of the pads

The transient oil film force of each pad is shown in Fig. 12. With the whirling of the shaft, the transient oil film force changes periodically. The average values of transient oil film force of the pad No. 0,1 , 2,3 and 4 are $269,354,417,343$ and $263 \mathrm{~N}$, respectively. The pad No. 2 mainly acts as a load pad, providing an oil film force to support the shaft. The rotations of the pads No. $0,1,3$ and 4 mainly regulate the oil film force, making the whirl center near the $\mathrm{x}$ axis. When the shaft is on the right side of the whirl orbit, the pads No. 3 and No. 4 play a dominant role. When the shaft is on the left side of the whirl orbit, the pads No. 0 and No. 1 play a dominant role.

When the position of the shaft changes, the pads rotate accordingly, and the flexible rotation of the pads is conducive to the formation of the wedge required for the hydrodynamic pressure. The Different rotation angles, directions and different functions of the pads make the maximum transient force acting on the shaft in the $\mathrm{x}$ and $\mathrm{y}$ direction appears before the maximum position (the vertexes of the whirl orbit) of the shaft center in the $\mathrm{x}$ and $\mathrm{y}$ direction. Under the joint action of each pad, the whirl of the shaft is restrained, and the stability of the TPJBs is improved.

\section{CONCLUSION}

The main conclusions are as follows:

1) A $3 D$ transient hydrodynamic lubrication model of TPJBs is established based on the self-developed dynamic grid program and CFD method. In this model, the fluid-structure interaction between the flow field and the rotor motion, pads rotations was realized. The feasibility of the mod-el is proved by comparing with the experimental data.

2) With an increase in the unbalances, the whirling orbits enlarge and the bearing stability decreases.
Compared with fixed-pad bearings, the size of the shaft whirl orbit of the TPJBs is smaller. The whirl center is near the $y$-axis, but the whirl center drops obviously in the y-axis direction.

3) The results of different loading modes show that the center of the whirl orbit drop slightly and the size of the whirl orbit is smaller under LBP. This indicates that the LBP mode of TPJBs has higher stability margin.

4) During the whirling process of the shaft, the force acting on the shaft, the rotation angle of the pad and the oil film force of the pad change periodically. The period and frequency of these changes are the same as that of the shaft rotation. Different rotation angles, directions and different functions of the pads make the maximum force acting on the shaft appear before the maximum shaft center position. The whirl of the shaft is restrained and the stability of TPJBs is improved.

\section{ACKNOWLEDGEMENTS}

This work was supported by the National Natural Science Foundation of China (51506225), the General Program of Natural Science Foundation of Shandong Province (ZR2020ME174), the Key Research and Development Program of Shandong, China (2018GHY115018), the Fundamental Research Funds for the Central Universities (18CX02129A), and the Graduate Student Innovation Project, China University of Petroleum (East China) (YCX2019040).

\section{REFERENCES}

Armentrout, R. W., M. H. He, T. Haykin and A. E. Reed (2017). Analysis of turbulence and 
convective inertia in a water-lubricated tiltingpad journal bearing using conventional and CFD approaches. Tribology Transactions 60(6), 1129-1147.

Ding, A. S., X. D. Ren, X. S. Li and C. W. Gu (2018). Friction power analysis and improvement for a tilting-pad journal bearing considering air entrainment. Applied Thermal Engineering 145, 763-771.

EI Kayar, A., E. A. Salem, M. F. Khalil and A. A. Hegazy (1983). Two-dimensional finite difference solution for externally pressurized journal bearings of finite length. Wear 84(1), 113.

Gertzos, K. P., P. G. Nikolakopoulos and C. A. Papadopoulo (2008). CFD analysis of journal bearing hydrodynamic lubrication by Bingham lubricant. Tribology International 41(12), 1190-1204.

Ha, H. C., H. J. Kim and K. W. Kim (1994). Inlet pressure effects on the thermohydroclynamjc performance of a large tilting pad journal bearing. Journal of Tribology 177, 160-165.

Hagemannn, T., C. Zeh and H. Schwarze (2019). Heat convection coefficients of a tilting-pad journal bearing with directed lubrication. Tribology International 136, 114-126.

Lahmar, M. and B. Bou-Said (2008). Couple Stress Effects on the Dynamic Behavior of Connecting Rod Bearings in Both Gasoline and Diesel Engines. Tribology Transactions 51(1), 44-56.

Lai, T. W., Y. Hou, L. Niu, Y. Q. Zheng and S. T. Chen (2014). Numerical study on the load direction effect on the performance of tilting pad-journal gas bearing. Journal of Advanced Mechanical Design, Systems and Manufacturing 8(3).

Lee, D., K. H. Sun, B. Kim and D. Kang (2018). Thermal behavior of a worn tilting pad journal bearing: thermohydrodynamic analysis and pad temperature measurement. Tribology Transactions 61(6), 1074-1083.

Li, M. X., C. H. Gu, X. H. Pan, S. Y. Zheng and Q. $\mathrm{Li}$ (2016). A new dynamic mesh algorithm for studying the $3 \mathrm{D}$ transient flow field of tilting pad journal bearings. Proceedings of the Institution of Mechanical Engineers 230(12), 1470-1482.

Li, M. X., J. Wu, S. Y. Zheng, G. Y. Ying and S. L. Liu (2017b). Stability of bearing-rotor system with different bearing structures. Journal of Zhejiang University (Engineering Science) 51(11), 2239-2248.

Li, Q., S. L. Liu., X. H. Pan and S. Y. Zheng (2012). A new method for studying the $3 \mathrm{D}$ transient flow of misaligned journal bearings in flexible rotor-bearing systems. Journal of Zhejiang University: Science A 13(4), 293-310.

Li, Q., G. C. Yu, S. L. Liu and S. Y. Zheng (2012). Application of computational fluid dynamics and fluid structure interaction techniques for calculating the 3D transient flow of journal bearings coupled with rotor systems. Chinese Journal of Mechanical Engineering (English Edition) 25(5), 926-932.

Li, Q., S. Zhang, L. Ma, W. W. Xu and S. Y. Zheng (2017a). Stiffness and damping coefficients for journal bearing using the 3D transient flow calculation. Journal of Mechanical Science and Technology 31(5), 2083-2091.

Li, Q., S. Zhang, Y. J. Wang, W. W. Xu and Z. B. Wang (2019). Coupling mechanism analysis between shaft whirling and transient oil film force of journal bearings under dynamical load. Journal of Vibration and Shock 38(24), 158164.

Li, X. K., D. R. Gwynllyw, A. R. Davies and T. N. Phillips (2000). On the influence of lubricant properties on the dynamics of two-dimensional journal bearings. Journal of Non-Newtonian Fluid Mechanics 91(1), 29-59.

Marx, N., L. Fernandez, F. Barcelo and H. Spikes (2018). Shear thinning and hydrodynamic friction of viscosity modifier-containing oils. Part I: Shear thinning behavior. Tribology Letters 66(3).

Marx, N., L. Fernandez, F. Barcelo and H. Spikes (2018). Shear thinning and hydrodynamic friction of viscosity modifier-containing oils. Part II: Impact of shear thinning on journal bearing friction. Tribology Letters 66(3).

Parovay, E. F. and S. V. Falaleev (2017, September), CFD Analysis of a starved four-pad tilting-pad journal bearing with an elastic support of pads. IOP Conference Series: Materials Science and Engineering, Samara Univ, Semera, Russia, 302(1).

Raud, X., M. Fillon and M. Helene (2013). Numerical modelling of hydrostatic lift pockets in hydrodynamic journal bearings-Application to low speed working conditions of loading tilting pad journal bearings. Mechanics and Industry 14(5), 327-334.

Reynolds, O. (1886). On the theory of lubrication and its application to Mr. Beauchamp Tower's experiments, including an experimental determination of the viscosity of olive oil. Proceedings of the Royal Society of London 40, 191-203.

Roy, L. and S. K. Laha (2009). Steady state and dynamic characteristics of axial grooved journal 
Q. Li et al. / JAFM, Vol. 14, No. 6, pp. 1827-1837, 2021.

bearings. Tribology International 42(5), 754761.

Singhal, A. K., M. M. Athavale, H. Y. Li and Y. Jiang (2002). Mathematical basis and validation of the full cavitation model, Journal of Fluids Engineering 124(3), 617-624.

Taniguchi, S., T. Makino, K. Takeshita and T. Ichimura (1990). A thermohydrodynamic analysis of large tilting-pad journal bearing in laminar and turbulent flow regimes with mixing. Journal of Tribology 112(3), 542-548.

Tong, X. M. and A. Palazzolo (2018). Measurement and prediction of the journal circumferential temperature distribution for the rotordynamic morton effect. Journal of Tribology 140(3).

Tower, B. (1883). First report on friction experiments. Proceedings of the Institution of Mechanical Engineers 35(1), 29-35.

Varela, A. C. B. B. Nielsen and I. F. Santos (2013). Steady state characteristics of a tilting pad journal bearing with controllable lubrication: Comparison between theoretical and experimental results. Tribology International 58, 85-97.

Wang, Y. L. and Liu. Z. S (2011, August). Study on tilting characteristic of pads in tilting pad bearing. Proceedings of 2011 International Conference on Electronic and Mechanical Engineering and Information Technology, Harbin, Heilongjiang, China, 9, 4653-4657.

Yang, J. and A. Palazzolo (2021). Computational fluid dynamics based mixing prediction for tilt pad journal bearing TEHD modeling-part I: TEHD-CFD model validation and improvements. Journal of Tribology 143(1).

Yang, J. and A. Palazzolo (2021). Computational fluid dynamics based mixing prediction for tilt pad journal bearing TEHD modeling-part II: implementation with machine learning. Journal of Tribology 143(1).

Zheng, T. and N. Hasebe (2000). Calculation of equilibrium position and dynamic coefficients of a journal bearing using free boundary theory. Journal of tribology 122(3), 616-621. 